\title{
Identification of molecular pathway changes after spinal cord injury by microarray analysis
}

\author{
Haocong Zhang and Yan Wang*
}

\begin{abstract}
Background: Spinal cord injury (SCl) is highly related to the devastating sensory and motor dysfunction.

Methods: The GSE45006 gene expression profile dataset was downloaded from Gene Expression Omnibus, which was collected from 24 rats including 20 animals with injured T7 spinal cords using an aneurysm clip impact-compression injury model and killed after 1 day, 3 days, 1 week, 2 weeks, and 8 weeks and four sham-operated rats. Differentially expressed genes (DEGs) between the injured rats at each time point and the sham-operated rats were screened. DEGs commonly detected throughout different time points were further identified, followed by comparing the expression level of these DEGs at each time point between the injured spinal cord samples and controls. Pathway enrichment analysis of the common DEGs was performed.

Results: The difference in the expression level of 416 common DEGs was significant between the injured spinal cord samples and the controls at each time point $(P<0.05)$, with the most significant difference 1 day after $\mathrm{SCl}$. The common DEGs were enriched in three pathways, namely Fcy R-mediated phagocytosis, mitogen-activated protein kinase (MAPK) signaling pathway, and chemokine signaling pathway. AKT3 and RAC2 were enriched in all the three pathways; RAP1B in both MAPK signaling pathway and chemokine signaling pathway; and VAV1, $L Y N$, and HCK in both FCY R-mediated phagocytosis and chemokine signaling pathway.

Conclusions: This study has confirmed the occurrence of neuronal death, inflammation, and neuronal regeneration after SCI. AKT3, RAC2, VAV1, RAP18, LYN, and HCK may have critical roles in the pathological responses to SCI.
\end{abstract}

Keywords: Differentially expressed genes, Interaction network, Pathway enrichment analysis, Spinal cord injury

\section{Background}

Spinal cord injury (SCI) can lead to sensory and motor dysfunction, manifesting a wide range of functional deficits depending on the level of injury [1, 2]. Following the mechanical injury that rapidly kills neurons and glia at the initial injury site, devastating and delayed secondary cellular and molecular events occur. These include enlarged cell death through necrosis and apoptosis, which is exacerbated by the presence of inflammatory cells, such as neutrophils, macrophages, and T cells $[3,4]$. Among these inflammatory cells, macrophages, a type of phagocyte, have a dominant role, which not only damage neurons and

\footnotetext{
* Correspondence: yanwang4157@163.com

Department of Orthopaedics, The General Hospital of PLA, No. 28 Fuxing Road, Haidian District, Beijing 100853, China
}

glia but also are essential for the reconstruction of injured tissues by phagocytosis [5]. Chemokines, a type of small chemoattractant peptide, which can provide directional cues for the cell trafficking, are also necessary for the inflammatory responses [6]. Microarray analysis has been proven reliable and efficient in elucidating the molecular events following SCI [7-9]. For example, microarray analysis together with immunohistochemical studies has validated neuronal regeneration after SCI based upon the up-regulation of neurite growth and regeneration-associated genes after SCI, such as those encoding VGF, SPRR1A, and GAP43 [10-12]. Chamankhah et al. have identified gene expression profiles at day 1 , day 3 , week 1 , week 2 , and week 8 in rats following SCI, and they have found that the adaptive and induced innate immune 
responses span the acute and subacute phases and also persist throughout the chronic phase of SCI, despite the induced innate responses are more active during the acute phase, while the adaptive immune response processes are more significant during the chronic phase [13].

In the present study, the GSE45006 microarray data submitted by Chamankhah et al. that were collected from rats at different time points after SCI were downloaded and further analyzed. Here, we identified differentially expressed genes (DEGs) that were common throughout different time points. Then, based on the common DEGs, pathway enrichment analysis was performed and an interaction network was also constructed. These common DEGs may be essential for the pathological responses in rats after $\mathrm{SCI}$, thus can be alternatives for screening new therapeutic targets for spinal cord-injured patients.

\section{Methods \\ Microarray data}

Gene expression profile dataset GSE45006 was downloaded from the Gene Expression Omnibus (GEO, http://www.ncbi.nlm.nih.gov/geo/) database. The annotation platform was GPL1355 [Rat230_2] Affymetrix Rat Genome 2302.0 Array. As described by Chamankhah et al., a total of 24 animals were used and divided into six groups of four animals each. Twenty female Wistar rats in five groups underwent a T6-T8 laminectomy and then received a 35-g (Walsh) moderate to severe aneurysm clip impact-compression injury at T7 for $1 \mathrm{~min}$, which were killed after 1 day, 3 days, 1 week, 2 weeks, and 8 weeks following the surgery. For each operated animal, a tissue sample was collected from the T7 spinal cord epicenter for extraction of total RNA; meanwhile, the samples were also collected from the T7 spinal cord tissue of sham-operated rats in the control group.

\section{Microarray data preprocessing and screening of DEGs}

All the hybridized probes were submitted to the annotation platform to obtain gene names, and null probes were removed. Then, the expression profile data of the resulting genes were subjected to $\log 2$ conversion [14].

Genes with $\mid \log 2 \mathrm{FC}$ (fold change) $\mid>1$ and a false discovery rate (FDR) value $<0.05$ were selected as DEGs [15]. The FDR value was obtained by adjusting the raw $P$ values with the BH (Benjamin-Hochberg) method [16].

\section{Further comparison of common DEGs}

The common DEGs throughout different time points were further screened, which were defined as successive DEGs. Then, $t$ test was used to determine whether there was significant difference in the expression level of these common DEGs between the injured spinal cord samples and the controls at each time point $(P<0.05)$ [17].

\section{Pathway enrichment analysis of the common DEGs}

Database for Annotation, Visualization and Integrated Discovery (DAVID, https://david.ncifcrf.gov/home.jsp) bioinformatics resources consist of an integrated biological knowledgebase and analytic tools aimed at systematically extracting biological meaning from large gene or protein lists [18]. The Gene Functional Classification Tool of DAVID was used to perform pathway enrichment analysis based on the Kyoto Encyclopedia of Genes and Genomes (KEGG) database using a gene-set approach to determine whether there is a functional change of a set of genes $(P<0.05)[19,20]$.

\section{Construction of interaction network based on pathway}

Search Tool for the Retrieval of Interacting Genes/ Proteins (STRING) can provide uniquely comprehensive coverage and ease of access to both experimental as well as predicted interaction information that is denoted with a confidence score [21]. In the present study, STRING was used to construct an interaction network of the DEGs significantly enriched in pathways, and a score threshold of 0.4 was adopted. The resulting network was visualized using Cytoscape [22].

\section{Results}

\section{DEGs screened at each time point after SCI}

In total, 19,763 genes were obtained for DEG screening after removing null probes. A total of 3405, 2165, 1262, 1391, and 1841 DEGs were obtained at day 1, day 3 , week 1 , week 2 , and week 8 following SCI, respectively.

Further comparison of the expression of the common DEGs A total of 416 DEGs were found common throughout the five different time points, such as the up-regulated ones, RAC2, RAP1B, VAV1, LYN, HCK, JUN, and the down-regulated ones, $A K T 3$ (Table 1). The result of the $t$ test revealed that there was significant difference in the expression level of these common DEGs between the injured rats at each time point and the sham-operated ones $(P<0.05)$, with the most significant difference 1 day after SCI $(P=1.42 \mathrm{e}-05)$, followed by less significant difference, 3 days $(P=0.000115)$, 1 week $(P=0.0001974)$, 8 weeks $(P=0.006143)$, and 2 weeks $(P=0.005989)$, after SCI successively (Fig. 1).

\section{Pathway enrichment analysis of the common DEGs}

According to the pathway enrichment analysis, the 416 common DEGs were enriched in three pathways (Table 1), that is, Fcy R-mediated phagocytosis pathway $(P=4.367 \mathrm{e}-04)$, mitogen-activated protein kinase 
Table 1 Some of the genes successively differentially expressed across different time points

\begin{tabular}{|c|c|c|c|c|c|c|c|c|c|c|}
\hline \multirow[b]{2}{*}{ Gene symbol } & \multicolumn{2}{|l|}{ On day 1} & \multicolumn{2}{|l|}{ On day 3} & \multicolumn{2}{|l|}{ At week 1} & \multicolumn{2}{|l|}{ At week 2} & \multicolumn{2}{|l|}{ At week 8} \\
\hline & $\log F C$ & adj. P val & $\log F C$ & adj. P val & $\log F C$ & adj. P val & $\log F C$ & adj. P val & $\log F C$ & adj. P val \\
\hline Akt3 & -2.9097 & 0.000187 & -1.96743 & 0.000164 & -2.19211 & 0.002497 & -2.92584 & $1.42 \mathrm{E}-06$ & -2.42503 & $6.64 \mathrm{E}-05$ \\
\hline Jun & 1.524331 & 0.000472 & 1.83288 & 0.00021 & 1.795763 & 0.002161 & 0.002161 & 1.795763 & 1.092874 & 0.00173 \\
\hline Rap1b & 1.592105 & 0.000158 & 1.131165 & 0.00048 & 1.546159 & 0.004442 & 1.2095 & $3.48 \mathrm{E}-05$ & 1.285494 & 0.000403 \\
\hline Lyn & 4.070571 & 0.000472 & 2.399984 & 0.00572 & 3.612514 & 0.006097 & 3.195225 & 0.000343 & 2.950347 & 0.00239 \\
\hline Rac2 & 4.305635 & 0.000242 & 2.226036 & 0.00487 & 3.706749 & 0.005252 & 2.863293 & 0.000298 & 2.376156 & 0.00531 \\
\hline Vav1 & 5.353884 & 3.86E-05 & 2.458724 & 0.00195 & 4.618522 & 0.003165 & 3.970052 & $1.08 \mathrm{E}-05$ & 4.247292 & 8.95E-05 \\
\hline Hck & 7.363526 & $5.02 \mathrm{E}-06$ & 5.202298 & $4.22 \mathrm{E}-05$ & 6.446867 & 0.001662 & 5.930991 & 7.95E-07 & 6.162797 & $1.23 \mathrm{E}-05$ \\
\hline
\end{tabular}

(MAPK) signaling pathway $(P=0.001434)$, and chemokine signaling pathway $(P=0.001592)$.

\section{Interaction of DEGs significantly enriched in the three pathways}

According to the constructed interaction network of DEGs significantly enriched in the three pathways, $A K T 3$ (serine/threonine protein kinase 3) and RAC2 (Ras-related C3 botulinum toxin substrate 2) were enriched in all the three pathways (Fig. 2); Rap1B (Rasrelated protein RAP1B) was involved in both the MAPK signaling pathway and the chemokine signaling pathway; VAV1 (guanine nucleotide exchange factor) and two Src family members, $L Y N$ (tyrosine protein kinase) and $H C K$ (hemopoietic cell kinase), were involved in both the $\mathrm{Fc \gamma}$ $\mathrm{R}$-mediated phagocytosis and the chemokine signaling pathway (Fig. 3).

\section{Discussion}

Chamankhah et al. have performed both time point and time-series analyses of the identified DEGs, which were

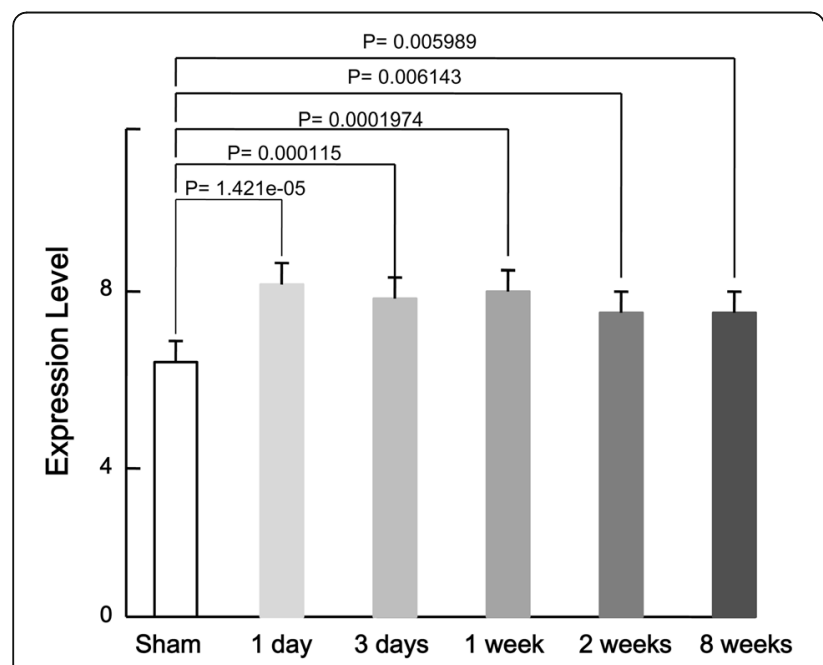

Fig. 1 Further comparison of the expression levels of the common DEGs between the injured spinal cord samples at 1 day, 3 days, 1 week, 2 weeks, and 8 weeks after $\mathrm{SCl}$ and the control successively further analyzed using Gene Ontology (GO) [13]. In the present study, after we identified genes differentially expressed at each time point, only those expressed successively across different time points were further studied by investigating the pathways these genes were involved in, through which it is found that the common DEGs were significantly enriched in three pathways, namely $\mathrm{Fc} \gamma \mathrm{R}$-mediated phagocytosis pathway, MAPK signaling pathway, and chemokine signaling pathway, and may have a profound implication for the pathological responses in rats at the cellular and molecular level after SCI. The chemokine signaling pathway is transduced by chemokine receptors (G protein-coupled receptors) expressed on the immune cells, which mediate the activation-diverse downstream pathways resulting in cellular polarization and actin reorganization [6]. The Fcy R-mediated phagocytosis is mediated by the Fcy receptors (Fc receptor for immunoglobulin G) [23]. Despite phagocytosis being universally acknowledged after

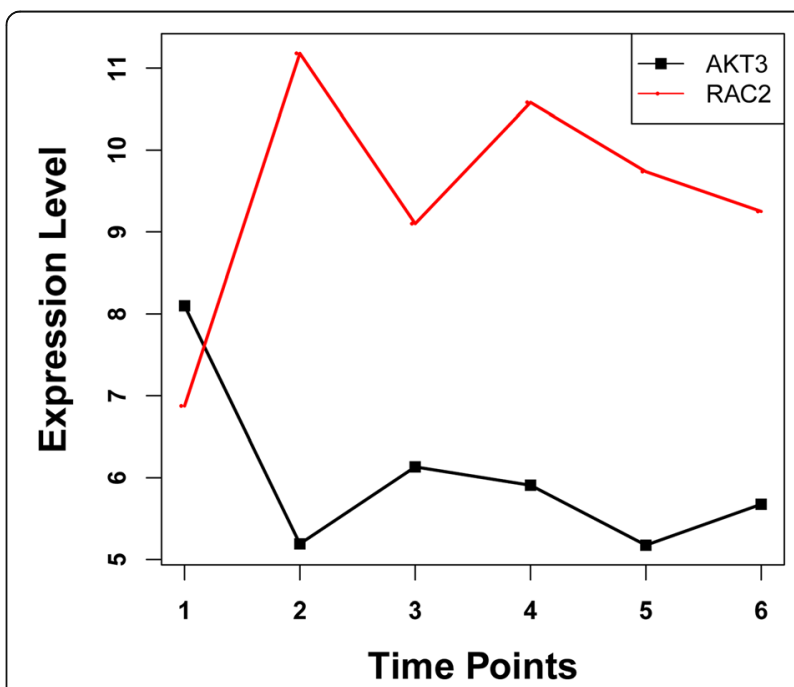

Fig. 2 The expression profile of $A K T 3$ and $R A C 2$ over time after spinal cord injury. The numbers 2, 3, 4, 5, and 6 on the horizontal ordinate represent 1 day, 3 days, 1 week, 2 weeks, and 8 weeks after $\mathrm{SCl}$, respectively, and the first point of each line represents the expression level of the corresponding gene in the control 


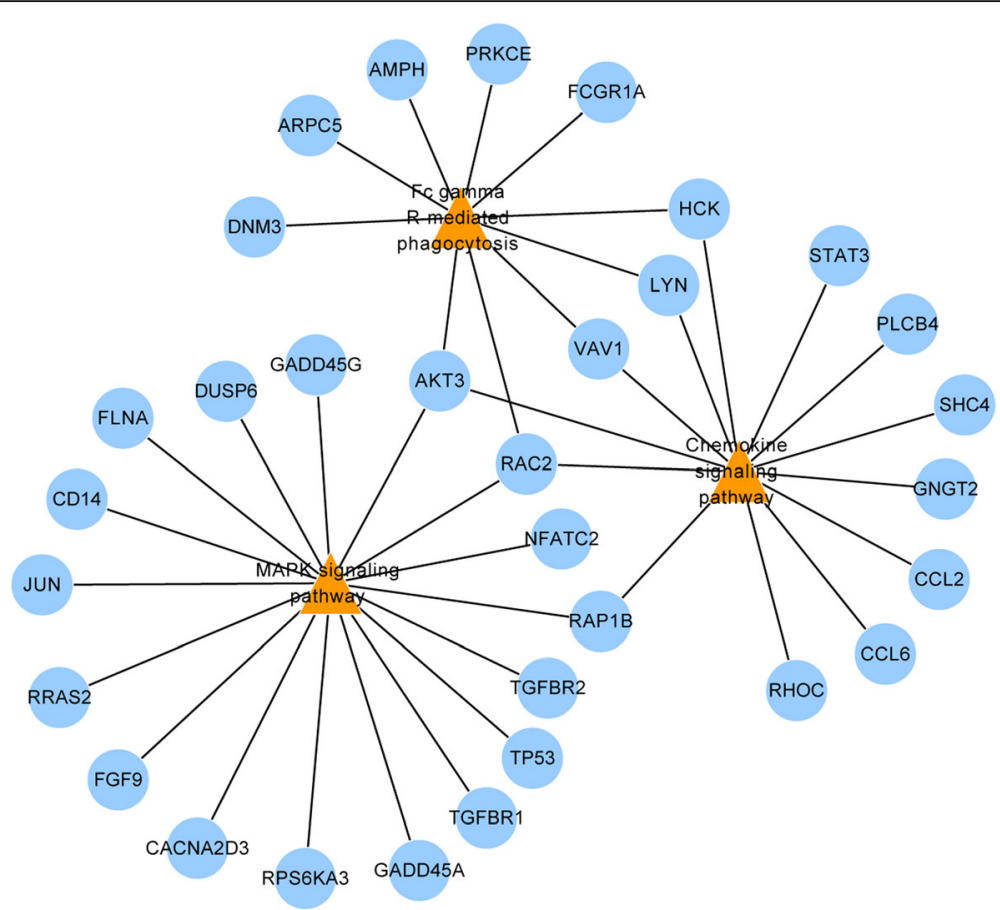

Fig. 3 Interaction of DEGs significantly enriched in the three pathways. The orange triangles represent the three enriched pathways; the blue circles represent the DEGs enriched in the pathways

SCI [5, 24], Fcy R-mediated phagocytosis is seldom reported in spinal cord-injured rats. These two enrichment pathways further confirmed the key role of inflammation after SCI, conforming with numerous previous studies $[3,4,25,26]$ that have demonstrated the involvement of two major types of inflammatory cells within certain time periods after SCI. For the MAPK cascade pathway, it is a highly conserved module that is involved in various cellular functions. Two MAPKs, ERK1/2 (extracellular signal-related kinases) and p38 MAPK (p38alpha/ beta/gamma/delta), mediate inducible nitric oxide synthase (iNOS)-induced spinal neuron degeneration, which is a general way to cause neuronal apoptosis by generating nitric oxide (NO) after acute traumatic SCI [27].

$A K T 3$ and $R A C 2$ were enriched in all the three pathways, indicating their important role after SCI. The AKT (also called protein kinase B) family consists of three members, Akt1/PKB $\alpha$, Akt2/PKB $\beta$, and Akt3/PKB , which share a high degree of structural similarity [28]. Peviani et al. have proposed that down-regulation or lack of induction of the P13K/AKT prosurvival pathway may be responsible for the defective response of spinal cord motor neurons to injury and their consequent cell death [29]. The finding that $A K T 3$ is significantly down-regulated across all the time points overall in our study further validates their proposal.

The Rho guanosine triphosphatases (GTPases) comprising RAC1 and RAC2 are key regulators of cytoskeletal dynamics and affect many cellular processes, including cell polarity, migration, vesicle trafficking, and cytokinesis [30]. Overall, the expression of $R A C 2$ was up-regulated after $\mathrm{SCI}$ in our study, which is consistent with the finding that Rho activity is raised after SCI [31]. Activation of Rac GTPase has been proven to be involved in the p75 neurotrophin receptor-dependent apoptosis [31, 32]. Furthermore, Harrington et al. have reported that this apoptosis pattern is mediated by activation of c-jun $\mathrm{N}$ terminal kinase [32], which can phosphorylate c-JUN, a major component of the JUN family protein (c-JUN, JUNB, and JUND) members that are necessary for the assembly of the AP-1 transcription factor complex [33]. c-JUN, involved in the MAPK signaling pathway, is highly induced in response to neuronal injury [34, 35], which is consistent with the up-regulation of its expression observed in our study.

Additionally, VAV1, $L Y N$, and $H C K$ were involved in both the Fcy R-mediated phagocytosis and the chemokine signaling pathway. $V A V 1$ is engaged in the regulation of T cell migration [36], and the up-regulation of its expression proves the participation of $\mathrm{T}$ cells in immune response after SCI. The expression of two Src family members, $H C K$ and $L Y N$, with similar roles in a signal pathway, were both up-regulated in our study, which is consistent with the up-regulation of $L Y N$ observed by Yang et al., who also studied the peripheral nerve injuryinduced modification of genes in Sprague-Dawley rat 
dorsal spinal cords using both microarray analysis and immunohistochemistry [9]. Meanwhile, RAP1B (Rasrelated protein Rap-1B) was also observed to participate in both the MAPK signaling pathway and the chemokine signaling pathway. Schwamborn and Püschel have discovered that localization of the GTPase RAP1B (Rasrelated protein Rap-1B) to the tip of a single neurite is a decisive step in determining which neurite becomes the axon [37]; thus, the up-regulation of RAP1B expression in our study may suggest the occurrence of neuronal regeneration after SCI.

\section{Conclusions}

In summary, we identified genes that were differentially expressed at different time points after SCI in rats, as well as three pathways, Fcy R-mediated phagocytosis pathway, MAPK signaling pathway, and chemokine signaling pathway, through which these DEGs were speculated to respond to SCI. This further proves that neuronal death, inflammation, and neuronal regeneration occur after SCI, especially inflammation. And, DEGs, $A K T 3, R A C 2, V A V 1$, $R A P 1 B, H C K$, and $L Y N$, that are involved in the above three pathways may be essential for the pathological responses to $\mathrm{SCI}$, which may be considered as candidate genes for the targeted therapy of SCI. Our work has deepened our understandings into the molecular mechanisms underlying SCI. However, since our findings here were obtained by bioinformatics methods, they should be taken prudently and further validated by experimental proofs before any clinical use.

\section{Abbreviations \\ AKT3: Serine/threonine protein kinase 3; $\mathrm{BH}$ : Benjamin-Hochberg; DAVID: Database for Annotation, Visualization and Integrated Discovery; DEGs: Differentially expressed genes; FDR: False discovery rate; GEO: Gene Expression Omnibus; GO: Gene Ontology; GTPases: Guanosine triphosphatases; HCK: Hemopoietic cell kinase; iNOS: Inducible nitric oxide synthase; KEGG: Kyoto Encyclopedia of Genes and Genomes; LYN: Tyrosine protein kinase; NO: Nitric oxide; RAC2: Ras-related C3 botulinum toxin substrate 2; Rap1B: Ras-related protein RAP1B; RAP1B: Ras-related protein Rap-1B; SCl: Spinal cord injury; STRING: Search Tool for the Retrieval of Interacting Genes/Proteins; VAV1: Guanine nucleotide exchange factor}

\section{Acknowledgements}

None.

\section{Funding}

None.

\section{Availability of data and materials}

The dataset "Gene expression profile dataset GSE45006" supporting the conclusions of this article can be downloaded from the GEO (Gene Expression Omnibus, http://www.ncbi.n/m.nih.gov/geo/) database. The annotation platform was GPL1355 [Rat230_2] Affymetrix Rat Genome 230 2.0 Array.

\section{Authors' contributions}

$\mathrm{HZ}$ participated in the design of this study. $\mathrm{HZ}$ and $\mathrm{YW}$ performed the statistical analysis. YW collected the important background information and drafted the manuscript. $\mathrm{HZ}$ helped to draft the manuscript. Both authors read and approved the final manuscript.

\section{Competing interests}

The authors declare that they have no competing interests.

\section{Consent for publication}

Not applicable.

Ethics approval and consent to participate

None.

Received: 23 June 2016 Accepted: 5 September 2016

Published online: 15 September 2016

References

1. Schwab ME, Bartholdi D. Degeneration and regeneration of axons in the lesioned spinal cord. Physiol Rev. 1996;76:319-70.

2. Hulsebosch CE. Recent advances in pathophysiology and treatment of spinal cord injury. Adv Physiol Educ. 2002;26:238-55.

3. Bethea JR, Dietrich DW. Targeting the host inflammatory response in traumatic spinal cord injury. Curr Opin Neurol. 2002;15:355-60.

4. Schnell $L$, Fearn $S$, Klassen $H$, Schwab ME, Perry VH. Acute inflammatory responses to mechanical lesions in the CNS: differences between brain and spinal cord. Eur J Neurosci. 1999:11:3648-58.

5. Popovich PG, Guan Z, Wei P, Huitinga I, van Rooijen N, Stokes BT. Depletion of hematogenous macrophages promotes partial hindlimb recovery and neuroanatomical repair after experimental spinal cord injury. Exp Neurol. 1999:158:351-65.

6. Zlotnik A, Yoshie O. Chemokines: a new classification system and their role in immunity. Immunity. 2000;12:121-7.

7. Pan JZ, Ni L, Sodhi A, Aguanno A, Young W, Hart RP. Cytokine activity contributes to induction of inflammatory cytokine mRNAs in spinal cord following contusion. J Neurosci Res. 2002;68:315-22.

8. HAYASHI M, UEYAMA T, NEMOTO K, TAMAKI T, SENBA E. Sequential mRNA expression for immediate early genes, cytokines, and neurotrophins in spinal cord injury. J Neurotrauma. 2000;17:203-18.

9. Yang L, Zhang FX, Huang F, Lu YJ, Li GD, Bao L, Xiao HS, Zhang X. Peripheral nerve injury induces trans-synaptic modification of channels, receptors and signal pathways in rat dorsal spinal cord. Eur J Neurosci. 2004;19:871-83.

10. Bonilla IE, Tanabe K, Strittmatter SM. Small proline-rich repeat protein $1 A$ is expressed by axotomized neurons and promotes axonal outgrowth. J Neurosci. 2002;22:1303-15.

11. Costigan M, Befort K, Karchewski L, Griffin RS, D'Urso D, Allchorne A, Sitarski J, Mannion JW, Pratt RE, Woolf CJ. Replicate high-density rat genome oligonucleotide microarrays reveal hundreds of regulated genes in the dorsal root ganglion after peripheral nerve injury. BMC Neurosci. 2002;3:16.

12. Kapfhammer JP, Schwab ME. Increased expression of the growth-associated protein GAP-43 in the myelin-free rat spinal cord. Eur J Neurosci. 1994;6: 403-11

13. Chamankhah M, Eftekharpour E, Karimi-Abdolrezaee S, Boutros PC, SanMarina S, Fehlings MG. Genome-wide gene expression profiling of stress response in a spinal cord clip compression injury model. BMC Genomics. 2013;14:583. doi:10.1186/1471-2164-14-583.

14. Fujita A, Sato JR, Rodrigues LO, Ferreira CE, Sogayar MC. Evaluating different methods of microarray data normalization. BMC Bioinformatics. 2006;7:469.

15. Gentleman R, Carey V, Huber W, Irizarry RA, Dudoit S. Bioinformatics and computational biology solutions using $\mathrm{R}$ and Bioconductor. New York: Springer; 2005.

16. Benjamini $Y$, Hochberg $Y$. Controlling the false discovery rate: a practical and powerful approach to multiple testing. J R Stat Soc Ser B Methodol. 1995:57:289-300

17. O'Connor J, Robertson E. Student's t-Test, MacTutor History of Mathematics Archive. University of St Andrews, http://www-history.mcs.st-andrews.ac.uk/ Biographies/Gosset.html. Accessed 2012.

18. Huang DWSB, Lempicki RA. Systematic and integrative analysis of large gene lists using DAVID Bioinformatics Resources. Nat Protoc. 2009;4:44-57.

19. Nam D, Kim S-Y. Gene-set approach for expression pattern analysis. Brief Bioinform. 2008:9:189-97.

20. Alvord G, Roayaei J, Stephens R, Baseler MW, Lane HC, Lempicki RA. The DAVID Gene Functional Classification Tool: a novel biological module-centric algorithm to functionally analyze large gene lists. Genome Biol. 2007;8:R183.

21. Szklarczyk D, Franceschini A, Kuhn M, Simonovic M, Roth A, Minguez $P$, Doerks T, Stark M, Muller J, Bork P. The STRING database in 2011: functional 
interaction networks of proteins, globally integrated and scored. Nucleic Acids Res. 2011;39:D561-8.

22. Smoot ME, Ono K, Ruscheinski J, Wang P-L, Ideker T. Cytoscape 2.8: new features for data integration and network visualization. Bioinformatics. 2011; 27:431-2.

23. Fridman WH. Fc receptors and immunoglobulin binding factors. FASEB J. 1991:5:2684-90

24. Greenhalgh AD, David S. Differences in the phagocytic response of microglia and peripheral macrophages after spinal cord injury and its effects on cell death. J Neurosci. 2014;34:6316-22. doi:10.1523/JNEUROSCI.4912-13.2014.

25. Carlson SL, Parrish ME, Springer JE, Doty K, Dossett L. Acute inflammatory response in spinal cord following impact injury. Exp Neurol. 1998;151:77-88.

26. Hausmann O. Post-traumatic inflammation following spinal cord injury. Spinal Cord. 2003;41:369-78.

27. Xu Z, Wang B-R, Wang X, Kuang F, Duan X-L, Jiao X-Y, Ju G. ERK1/2 and p38 mitogen-activated protein kinase mediate iNOS-induced spinal neuron degeneration after acute traumatic spinal cord injury. Life Sci. 2006;79:1895-905.

28. Kandel ES, Hay N. The regulation and activities of the multifunctional serine/ threonine kinase Akt/PKB. Exp Cell Res. 1999;253:210-29.

29. Peviani M, Cheroni C, Troglio F, Quarto M, Pelicci G, Bendotti C. Lack of changes in the PI3K/AKT survival pathway in the spinal cord motor neurons of a mouse model of familial amyotrophic lateral sclerosis. Mol Cell Neurosci. 2007;34:592-602

30. Wennerberg K, Der CJ. Rho-family GTPases: it's not only Rac and Rho (and I like it). J Cell Sci. 2004;117:1301-12.

31. Dubreuil $\mathrm{Cl}$, Winton MJ, McKerracher L. Rho activation patterns after spinal cord injury and the role of activated Rho in apoptosis in the central nervous system. J Cell Biol. 2003;162:233-43.

32. Harrington AW, Kim JY, Yoon SO. Activation of Rac GTPase by p75 is necessary for c-jun N-terminal kinase-mediated apoptosis. J Neurosci. 2002; 22:156-66.

33. Jochum W, Passegué E, Wagner EF. AP-1 in mouse development and tumorigenesis. Oncogene. 2001;20:2401-2412.

34. Raivich G, Bohatschek M, Da Costa C, Iwata O, Galiano M, Hristova M, Nateri AS, Makwana M, Riera-Sans L, Wolfer DP. The AP-1 transcription factor c-Jun is required for efficient axonal regeneration. Neuron. 2004;43:57-67.

35. Herdegen $\mathrm{T}$, Leah J. Inducible and constitutive transcription factors in the mammalian nervous system: control of gene expression by Jun, Fos and Krox, and CREB/ATF proteins. Brain Res Rev. 1998;28:370-490.

36. Pasquale EB. Eph-ephrin bidirectional signaling in physiology and disease. Cell. 2008;133:38-52.

37. Schwamborn JC, Püschel AW. The sequential activity of the GTPases Rap1B and Cdc42 determines neuronal polarity. Nat Neurosci. 2004;7:923-9.

\section{Submit your next manuscript to BioMed Central and we will help you at every step:}

- We accept pre-submission inquiries

- Our selector tool helps you to find the most relevant journal

- We provide round the clock customer support

- Convenient online submission

- Thorough peer review

- Inclusion in PubMed and all major indexing services

- Maximum visibility for your research

Submit your manuscript at www.biomedcentral.com/submit
Biomed Central 